\title{
Magnitude of pancreatic exocrine insufficiency and associated factors among adult diabetic patients attending Madda Walabu University Goba referral hospital, south east Ethiopia, 2019
}

Tadele Regasa Gemechu ( $\square$ tdlrgs104@gmail.com )

Madda Walabu university https://orcid.org/0000-0001-9278-4778

Minale Fekadie Baye

Jimma university

Gesese Bogale Awgichew

Jimma University

Behonegn Birhan Chekole

Jimma University

Zegeye Regassa Gemechu

Madda Walabu university

Habtamu Gezehagn Mulugeta

Madda Walabu university

Damtew Solomon Shiferaw

Madda Walabu university

Tesaka Wondimnew Damtew

Jimma University

Research article

Keywords: diabetes mellitus, pancreatic exocrine insufficiency, serum amylase, serum lipase.

Posted Date: August 27th, 2020

DOI: https://doi.org/10.21203/rs.3.rs-51768/v1

License: (c) (i) This work is licensed under a Creative Commons Attribution 4.0 International License.

Read Full License 


\section{Abstract}

Background: Diabetes mellitus is a group of metabolic disorders which is characterized by increased blood glucose level. It causes a lot of systemic complications including pancreatic atrophy that leads to pancreatic exocrine insufficiency. It is more common among type 1 diabetic than type 2 diabetic patients. The aim of this study was to assess magnitude of pancreatic exocrine insufficiency and associated factors among diabetic patients attending Madda Walabu University Goba referral hospital, 2019.

Materials and method: An institution based cross sectional study was conducted on 286 diabetic patients during study period. The study participants were selected by systematic random sampling technique among diabetic patients attending Madda Walabu University Goba referral hospital internal medicine department. The demographic data of the study participants were collected by face to face interview. Serum and urine samples were collected from each patient and analyzed to check pancreatic exocrine insufficiency and factors associated with it. The raw data were entered in to Epi Data V 3.0.2 and exported in to SPSS V 25 for analysis. Descriptive analysis and multiple logistic regression was done and variables with $95 \%$ confidence interval and p-value less than 0.05 were used to declare statistical significance.

Results: The study participants were 250 (87.4\%) type 2 diabetic and 36 (12.6\%) type 1 diabetic patients. Among them $21.3 \%$ suffered from pancreatic exocrine insufficiency. Amylase insufficiency found in $44.4 \%$ of type 1 and $16.0 \%$ in type 2 diabetics. But, lipase insufficiency was seen in $41.7 \%$ of type 1 and $16.0 \%$ in type 2 diabetics. The pancreatic exocrine insufficiency was higher among type $1(44.44 \%)$ than type 2 (18.0\%). Smoking habit, alcohol consumption, duration of disease, usage of hypoglycemic agents, ketosuria and type of diabetics were significantly associated with pancreatic exocrine insufficiency.

Conclusion: There was an alteration of pancreatic exocrine enzyme secretion among both type 1 and type 2 diabetic patients. The habit of alcohol consumption, smoking and increase in the duration of the disease intensify the pancreatic insufficiency.

\section{Introduction}

Diabetes mellitus (DM) is a collection of metabolic derangement manifested by hyperglycemia and it has typical systemic complications such as, cardiovascular disease, retinopathy, nephropathy and neuropathy (1). The pancreas has dual function that serves as both endocrine and exocrine glands. As endocrine gland, it produces insulin hormone which regulate blood glucose level and as an exocrine gland it releases digestive enzymes in to gut (2).

Pancreas' vital role in macronutrient digestion is to secrete digestive enzymes in to the duodenum. The pancreatic acinar cells committed to synthesis of proteolytic, amylolytic, lipolytic, and nuclease digestive enzymes. Neuropathic effect of diabetes mellitus halts this activity of pancreas, that resulted in pancreatic exocrine insufficiency (PEI) and additionally, lack of insulin causes acinar cell atrophy. Maldigestion of nutrients due to pancreatic exocrine insufficiency resulted in malnutrition (3). 
The prevalence of pancreatic exocrine insufficiency in DM is high, ranging from $26-44 \%$ and from $12-$ $20 \%$ in T1DM and T2DM respectively (4). Diabetic neuropathy is one of chronic diabetic complication that resulted in pancreatic exocrine insufficiency. Pancreatic exocrine insufficiency was reported in $52.4 \%$ of diabetes mellitus. T1DM patients were more suffered from PEI than T2DM with a magnitude of $51 \%$ and $32 \%$ respectively (5). Pancreatic cell death leads to altered pancreatic digestive enzyme synthesis. Accordingly, $28 \%$ of T2DM and all of them (100\%) had serum amylase activity $<23 \mathrm{U} / \mathrm{L}$ and serum lipase activity $<5 \mathrm{U} / \mathrm{L}$ which is below reference range. The decrement of amylase and lipase activity linked to altered exocrine pancreatic function (6).

Although a lot of study conducted on DM little was elaborated on diabetic pancreatic insufficiency. According to systematic review done in 2015 , PEI was more common in both type 1 and type 2 DM with a prevalence of (25-74\%) and (28-54\%) respectively (7). In Germany $51 \%$ of T1DM and 35\% of T2DM patients were suffered from PEI. As the duration of disease increases, the PEI also increases (8). Another clinical review conducted in 2011 on pancreas and DM relation indicated that $45-74 \%$ of T1DM and 28$36 \%$ of T2DM patients had exocrine pancreatic insufficiency (9).

The accumulation of fat in a pancreas due to metabolic syndrome (MetS) leads to pancreatic exocrine insufficiency. There was a reduction of Serum amylase activity in lean subjects as the progression of nonalcoholic fatty liver disease (NAFLD) (10). As the blood glucose level increases, the pancreatic capacity to secrete digestive enzyme decreases; resulting in maldigestion of carbohydrates, lipids and proteins. Therefore, analysis of serum amylase activity shows the progress of diabetic related complication (11). The prevalence of PEl in prediabetes alone and diabetic alone is $41 \%$ and $39 \%$ respectively. Not only this but also, newly diagnosed DM and prediabetes are suffered from PEI with a prevalence of $40 \%$. After acute pancreatitis, $39 \%$ of them suffered from PEI (12).

Even though sufficient studies were not available in Ethiopia on pancreatic exocrine insufficiency due to DM, it was estimated that in $2035,5.5 \%$ of Ethiopian population will suffer from DM. The increment of the diabetic patients from $4.4 \%$ in 2013 to $5.5 \%$ in 2035 needs a great attention to manage the complications of DM (13). So, the aim of this study was to assess the pancreatic exocrine insufficiency and associated factors among diabetic patients attending Madda Walabu University Goba referral hospital.

\section{Materials And Method}

A cross sectional study was conducted at Madda Walabu University Goba referral hospital (MWU GRH), Bale zone, South East Ethiopia which is $445 \mathrm{~km}$ far away from Addis Ababa from April 15 - May 30, 2019 on adult diabetic patients. All type 1 and type 2 diabetic patients of age 18 years and above visiting MWU Goba referral hospital were included. But diabetic patients those who were critically ill (in coma), diabetic patients with known pancreatic disease before DM onset and diabetic patients with salivary gland inflammation was excluded by card review and after physical inspection done by physician. 
The sample size was calculated by using a single population proportion formula by considering the following assumptions. Since the prevalence of pancreatic exocrine insufficiency among diabetics in Ethiopia was not available $P=50 \%$ and margin of error $d=0.05$ with $95 \%$ confidence interval was used.

Level of significance $\mathrm{a}=0.05 ; 95 \% \mathrm{Cl}: \mathrm{Za} /{ }_{2}=1.96$

$$
\begin{aligned}
& n=\frac{\left(\frac{z \alpha}{2}\right)^{2} p(1-p)}{d^{2}} \\
& n=\frac{(1.96)^{2} 0.5(1-0.5)}{0.05^{2}} \\
& \mathrm{n}=\underline{\mathbf{3 8 4}}
\end{aligned}
$$

Since source populations were less than 10,000 population correction formula was used as follow.

$n f=\frac{n}{1+\frac{n}{N}}$, where $\mathrm{n}=$ sample size calculated by single population formula, $\mathrm{N}=$ source population, $\mathrm{nf}=$ final sample size

$$
n f=\frac{384}{1+\frac{384}{869}}=\frac{384}{1.44}=267
$$

Expected non-response rate $=10 \%$

Non-response rate $=10 \% \star 267 \sim 27$

$\mathrm{Nf}=267+27=\underline{\mathbf{2 9 4}}$ Therefore, the total sample size of this study was 294.

The study unit was selected from the study population by systematic random sampling. There were 869 diabetic patients registered for attending MWU GRH for diabetic follow up and the sample size is 294 . Therefore, the sampling interval was:

$K=869 / 294=3$

The starting point was randomly selected among 1, 2 and 3; then those who registered every three intervals was participated in the study.

Interviewer-administered semi-structured questionnaire was adapted from different articles and guidelines those related to this study. Then the adapted tool was translated to the local languages Afan Oromo and Amharic languages and then back to English to ensure its consistency and accuracy. The questionnaire was contained socio-economic and demographic characteristic of the respondent, his/her health condition, life style and laboratory result registration format. The data collectors were trained on the objective of the study, data collection tool, approach to the interviewees, details of interviewing 
techniques, respect and maintaining privacy and confidentiality of the respondents before going to the data collection.

Data were collected by trained nurses by using a semi structured questionnaire through face to face interview and the type of DM and the type of medication they used were obtained by reviewing their card. The biological sample was collected and analyzed by the laboratory technologists according to standard operating procedure (SOP). Accordingly, $5 \mathrm{ml}$ of blood were collected from each study participants, then it was allowed to clot for 30 minute. Then, the cells were separated from serum by centrifuging the blood at $3000 \mathrm{rpm}$ for 5 minute. The serum sample was analyzed by spectrophotometer using different reagents for each parameter. From each study participants $10 \mathrm{ml}$ of urine was collected and analyzed after centrifugation at $3000 \mathrm{rpm}$ for 5 minute. The qualitative tests were done by immersing dry reagent dipstick in to urine and urine creatinine concentration and urine amylase activity was done by spectrophotometer.

Pancreatic exocrine insufficiency was the dependent variable and age, sex, residence, monthly income, occupation, and educational level were the socio economic independent variables. In addition to this blood pressure, type of DM, type of medication, age of onset, duration of the disease, smoking habit, alcohol use and BMI were included as life style and health condition independent variables. Serum analytes and calculated parameters such as FBS level, GFR, serum creatinine, serum urea and BUN were also included.

After checking the completeness of the data manually, the collected raw data were entered, cleaned and checked by Epi data version 4.0.2 and then it was exported to SPSS version 25 statistical packages for analysis. Descriptive analysis of different variables was done and variables with $p$-value $<0.25$ in binary logistic regression were used as a candidate for multiple logistic regression. Finally, the variables in multiple logistic regression that have significant association were identified by calculating odds ratio, with $95 \%$ confidence interval and p-value less than 0.05 was used to declare statistical significance.

To avoid the possibility of sampling bias, a minimum sample size sufficient to study the objectives of the study was determined. Pretest was done on $5 \%$ of the sample size (21 individuals) at Bale Robe Hospital. The collected data were checked for the completeness, clarity and consistency on the daily basis. Supervisor and principal investigator were closely follow both biological sample and information data collection process. After data collection, data were edited, coded, cleaned and some inconsistencies were checked in order to assess the quality of data.

Pancreatic exocrine insufficiency: is defined as inability of pancreas to secrete the digestive enzymes that result in maldigestion of macromolecules. It is manifested by either serum amylase activity $<28 \mathrm{U} / \mathrm{I}$ (normal range 28-100 U/I) or serum lipase activity <13 U/I (normal range 13-60 U/I) (14).

Five $\mathrm{ml}$ of venous blood was collected from each study participants in to tube with clot activator and the cells were separated from the serum within 30 minutes in order to prevent glycolysis that could be taken place by blood cells. Hemolysed sample was discarded because, it disturbs the absorbance of the 
solution during spectrophotometric reading. If the reading was above the measuring capacity of the spectrophotometer, the serum was diluted and the result was multiplied by dilution factor prior to reporting/registration (15).

Ethical clearance was obtained from institutional review board of Jimma University Institute of Health. Formal letter was obtained from Jimma University Institute of health to MWU GRH and the permission to conduct the study was obtained from chief executive of MWU GRH. Then both written and oral consent was obtained from each study participant. The name of the study participants was omitted from the questionnaire and the registration number/code was used to ensure the confidentiality. All abnormal laboratory result was linked to internal medicine department in the hospital for appropriate management of the study participants.

\section{Result}

Out of 294 diabetic patients selected to be participate in the study, 286 of them were took part in the study giving $97.28 \%$ response rate. The average age of diabetic onset of the study participants were 40.0 \pm 13.9 (95\% Cl: $38.4,41.6)$ years and the duration with DM was $8.8 \pm 7.0(95 \% \mathrm{Cl}: 8.0,9.7)$ years. T2DM patients had lower duration of disease ( $8.4 \pm 2.0$ years) than T1DM (11. \pm 6.2 years). T1DM had early diabetic onset ( $12.4 \pm 3.8$ years) than T2DM ( $44.0 \pm 9.7$ years).

Among 286 diabetic patients, 250 (87.4\%) of them were T2DM while T1DM accounts for 36 (12.6\%). Almost more than half $158(55.2 \%)$ of the study participants were only hypoglycemic agent users and about 63 (22.0\%) were used both hypoglycemic agents and insulin. Regarding family history of diabetics $86(30.1 \%)$ had family history of previous diabetics. Most of the study participants $195(68.2 \%)$ didn't use antihyperlipemic agents (refer Table 1). 
Table 1

Health condition of diabetic patients attending MWU GRH, South East Ethiopia, 2019.

\begin{tabular}{|c|c|c|c|}
\hline \multicolumn{2}{|l|}{ Variables } & \multicolumn{2}{|c|}{ Frequency } \\
\hline List & Category & Number & Percentage \\
\hline \multirow[t]{2}{*}{ Type of DM } & Type 1 & 36 & 12.6 \\
\hline & Type 2 & 250 & 87.4 \\
\hline \multirow[t]{4}{*}{ Use of DM medication } & Not on treatment & 23 & 8.0 \\
\hline & Insulin & 42 & 14.7 \\
\hline & hypoglycemic agent & 158 & 55.2 \\
\hline & both insulin and hypoglycemic agent & 63 & 22.0 \\
\hline \multirow[t]{2}{*}{ Family history of DM } & Yes & 86 & 30.1 \\
\hline & No & 200 & 69.9 \\
\hline \multirow[t]{2}{*}{ Use of antihyperlipemic agent } & Yes & 91 & 31.8 \\
\hline & No & 195 & 68.2 \\
\hline
\end{tabular}

\subsection{Laboratory profile of the study participants}

The mean fasting serum glucose level of diabetic patients was $364 \pm 116.6 \mathrm{mg} / \mathrm{dl}$ and $231.6 \pm$ $119.1 \mathrm{mg} / \mathrm{dl}$ among pancreatic insufficient and sufficient study subjects respectively and it was elevated among T1DM than T2DM. Their average serum amylase and lipase activity were also reduced in T1DM than T2DM in both those who developed PEI and who didn't develop PEI. Triglyceridemia was higher among diabetic patients who developed PEI than those who didn't developed. The mean serum triglyceride level of the study participants was $262.3 \mathrm{mg} / \mathrm{dl}, 243.0 \mathrm{mg} / \mathrm{dl}$ and $269.1 \mathrm{mg} / \mathrm{dl}$ among all diabetic patients, T1DM and T2DM suffering from PEI. Not only this but also their renal clearance was altered (Table 2).

The decrease in renal filtration ability as indicated by MDRD and EPI methods was reduced averagely to $86.54 \mathrm{ml} / \mathrm{min} / 1.73 \mathrm{~m}^{2}$ and $82.89 \mathrm{ml} / \mathrm{min} / 1.73 \mathrm{~m}^{2}$ respectively (Table 2 ). 
Table 2

Mean and standard deviations of laboratory profile of diabetic patients attending MWU GRH, Bale, South East Ethiopia, 2019.

\begin{tabular}{|c|c|c|c|c|c|c|}
\hline \multirow[t]{4}{*}{ Parameters } & \multirow{2}{*}{\multicolumn{2}{|c|}{$\begin{array}{l}\text { Overall patients }(n= \\
286) \\
\text { PEI }\end{array}$}} & \multicolumn{2}{|c|}{ Type 1 DM $(n=36)$} & \multicolumn{2}{|c|}{ Type 2 DM $(n=250)$} \\
\hline & & & PEI & & PEI & \\
\hline & $\begin{array}{l}\text { Yes }(n= \\
61)\end{array}$ & $\begin{array}{l}\text { No }(n= \\
225)\end{array}$ & $\begin{array}{l}\text { Yes }(n= \\
16)\end{array}$ & $\begin{array}{l}\text { No }(n= \\
20)\end{array}$ & $\begin{array}{l}\text { Yes }(n= \\
45)\end{array}$ & $\begin{array}{l}\text { No }(n= \\
205)\end{array}$ \\
\hline & $\begin{array}{l}\text { Mean } \pm \\
\text { SD }\end{array}$ & $\begin{array}{l}\text { Mean } \pm \\
\text { SD }\end{array}$ & $\begin{array}{l}\text { Mean } \pm \\
\text { SD }\end{array}$ & $\begin{array}{l}\text { Mean } \pm \\
\text { SD }\end{array}$ & $\begin{array}{l}\text { Mean } \pm \\
\text { SD }\end{array}$ & $\begin{array}{l}\text { Mean } \pm \\
\text { SD }\end{array}$ \\
\hline FBS (mg/dl) & $\begin{array}{l}364.4 \pm \\
116.6\end{array}$ & $\begin{array}{l}231.6 \pm \\
119.1\end{array}$ & $\begin{array}{l}392.6 \pm \\
109.4\end{array}$ & $\begin{array}{l}273.6 \pm \\
114.3\end{array}$ & $\begin{array}{l}354.4 \pm \\
118.6\end{array}$ & $\begin{array}{l}227.5 \pm \\
119.1\end{array}$ \\
\hline Amylase (u/l) & $\begin{array}{l}26.72 \pm \\
17.41\end{array}$ & $\begin{array}{l}62.28 \pm \\
47.12\end{array}$ & $\begin{array}{l}19.65 \pm \\
3.47\end{array}$ & $\begin{array}{l}54.81 \pm \\
51.15\end{array}$ & $\begin{array}{l}29.24 \pm \\
19.61\end{array}$ & $\begin{array}{l}63.01 \pm \\
46.77\end{array}$ \\
\hline Lipase (u/l) & $\begin{array}{l}11.76 \pm \\
7.94\end{array}$ & $\begin{array}{l}56.21 \pm \\
58.56\end{array}$ & $\begin{array}{l}10.88 \pm \\
5.69\end{array}$ & $\begin{array}{l}42.78 \pm \\
46.43\end{array}$ & $\begin{array}{l}12.07 \pm \\
8.63\end{array}$ & $\begin{array}{l}57.52 \pm \\
59.54\end{array}$ \\
\hline TG (mg/dl) & $\begin{array}{l}262.3 \pm \\
57.6\end{array}$ & $\begin{array}{l}257.1 \pm \\
63.2\end{array}$ & $\begin{array}{l}243.0 \pm \\
37.5\end{array}$ & $\begin{array}{l}236.2 \pm \\
52.6\end{array}$ & $\begin{array}{l}269.1 \pm \\
62.2\end{array}$ & $\begin{array}{l}259.1 \pm \\
63.8\end{array}$ \\
\hline Creatinine (mg/dl) & $\begin{array}{l}1.14 \pm \\
0.37\end{array}$ & $\begin{array}{l}1.16 \pm \\
0.46\end{array}$ & $\begin{array}{l}1.28 \pm \\
0.46\end{array}$ & $\begin{array}{l}1.05 \pm \\
0.42\end{array}$ & $\begin{array}{l}1.09 \pm \\
0.32\end{array}$ & $\begin{array}{l}1.17 \pm \\
0.46\end{array}$ \\
\hline Urea (mg/dl) & $\begin{array}{l}49.48 \pm \\
22.33\end{array}$ & $\begin{array}{l}46.41 \pm \\
18.94\end{array}$ & $\begin{array}{l}53.92 \pm \\
22.60\end{array}$ & $\begin{array}{l}42.08 \pm \\
16.74\end{array}$ & $\begin{array}{l}47.89 \pm \\
22.28\end{array}$ & $\begin{array}{l}46.83 \pm \\
19.13\end{array}$ \\
\hline BUN (mg/dl) & $\begin{array}{l}23.12 \pm \\
10.44\end{array}$ & $\begin{array}{l}21.67 \pm \\
8.85\end{array}$ & $\begin{array}{l}25.20 \pm \\
10.56\end{array}$ & $\begin{array}{l}19.73 \pm \\
7.85\end{array}$ & $\begin{array}{l}22.38 \pm \\
10.41\end{array}$ & $\begin{array}{l}21.88 \pm \\
8.94\end{array}$ \\
\hline BUN to CR ratio & $\begin{array}{l}21.82 \pm \\
10.60\end{array}$ & $\begin{array}{l}19.72 \pm \\
8.45\end{array}$ & $\begin{array}{l}19.80 \pm \\
5.18\end{array}$ & $\begin{array}{l}21.47 \pm \\
12.19\end{array}$ & $\begin{array}{l}22.54 \pm \\
11.92\end{array}$ & $\begin{array}{l}19.55 \pm \\
8.01\end{array}$ \\
\hline ACCR & $\begin{array}{l}2.30 \pm \\
0.64\end{array}$ & $\begin{array}{l}2.36 \pm \\
1.59\end{array}$ & $\begin{array}{l}2.44 \pm \\
0.59\end{array}$ & $\begin{array}{l}2.71 \pm \\
2.14\end{array}$ & $\begin{array}{l}2.24 \pm \\
0.66\end{array}$ & $\begin{array}{l}2.33 \pm \\
1.53\end{array}$ \\
\hline $\begin{array}{l}\text { GFR by MDRD } \\
\left(\mathrm{ml} / \mathrm{min} / 1.73 \mathrm{~m}^{2}\right)\end{array}$ & $\begin{array}{l}86.54 \pm \\
40.0\end{array}$ & $\begin{array}{l}95.47 \pm \\
53.35\end{array}$ & $\begin{array}{l}82.50 \pm \\
28.86\end{array}$ & $\begin{array}{l}128.25 \pm \\
86.9\end{array}$ & $\begin{array}{l}87.98 \pm \\
43.46\end{array}$ & $\begin{array}{l}92.27 \pm \\
48.02\end{array}$ \\
\hline $\begin{array}{l}\text { GFR by EPI } \\
\left(\mathrm{ml} / \mathrm{min} / 1.73 \mathrm{~m}^{2}\right)\end{array}$ & $\begin{array}{l}82.89 \pm \\
27.02\end{array}$ & $\begin{array}{l}89.86 \pm \\
32.74\end{array}$ & $\begin{array}{l}88.56 \pm \\
29.78\end{array}$ & $\begin{array}{l}115.15 \pm \\
45.2\end{array}$ & $\begin{array}{l}80.87 \pm \\
26.02\end{array}$ & $\begin{array}{l}87.39 \pm \\
30.29\end{array}$ \\
\hline
\end{tabular}

\subsection{Magnitude of pancreatic exocrine insufficiency}

Among 286 study participants, 61 (21.3\%) $(95 \% \mathrm{Cl}$ : 16.55, 26.05) of them were suffering from pancreatic exocrine impairment of either amylase or lipase. Serum amylase and lipase insufficiency were seen in 56 (19.6\%) (95\% Cl: 15.0, 24.2) and 55 (19.2\%) (95\% Cl: 14.64, 23.76) diabetic patients, respectively. 
Pancreatic exocrine impairment was more prevalent among T1DM 16 (44.44\%) (95\% Cl: 28.21, 60.67) than T2DM 45 (18.00\%) $(13.24,22.76)$ (Table 3).

Among 286 diabetic patients 56 (19.6\%) (95\% Cl: 15.0, 24.2) were found to have amylase insufficiency 44.4\% (95\% Cl: 28.21, 60.67) in type 1 and 16.0\% (95\% Cl: 11.46, 20.54) in type 2 diabetic patients. They also suffered from lipase insufficiency by a magnitude of $41.7 \%$ (95\% Cl: $25.59,57.81)$ and $16.0 \%(95 \%$ Cl: $11.46,20.54)$ among type 1 and type 2 DM, respectively. (Table 3 ).

Table 3

The magnitude of pancreatic exocrine insufficiency among diabetic patients attending MWU GRH, Bale, South East Ethiopia, 2019.

\begin{tabular}{|lllll|}
\hline Pancreatic enzymes insufficiency & & No. & $\%$ & $95 \%$ Cl \\
\hline Serum amylase insufficiency & Type 1 DM $(n=36)$ & 16 & 44.4 & $(28.21,60.67)$ \\
\cline { 2 - 5 } & Type 2 DM $(n=250)$ & 40 & 16.0 & $(11.46,20.54)$ \\
\cline { 2 - 5 } Serum lipase insufficiency & Total $(n=286)$ & 56 & 19.6 & $(15.0,24.2)$ \\
& Type 1 DM $(n=36)$ & 15 & 41.7 & $(25.59,57.81)$ \\
\cline { 2 - 5 } & Type 2 DM $(n=250)$ & 40 & 16.0 & $(11.46,20.54)$ \\
\cline { 2 - 5 } Pancreatic exocrine insufficiency (PEI) & Total $(n=286)$ & 55 & 19.2 & $(14.64,23.76)$ \\
\cline { 2 - 5 } & Type 1 DM $(n=36)$ & 16 & 44.4 & $(28.21,60.67)$ \\
\cline { 2 - 5 } & Type 2 DM $(n=250)$ & 45 & 18.0 & $(13.24,22.76)$ \\
\cline { 2 - 5 } & Total $(n=286)$ & 61 & 21.3 & $(16.55,26.05)$ \\
\hline
\end{tabular}

\subsection{Factors associated to pancreatic exocrine insufficiency}

Each independent variable entered in to binary logistic regression with pancreatic exocrine insufficiency as a dependent variable separately. Its result illustrated on the following table (Table 4). 
Table 4

binary logistic regression for factors associated to pancreatic exocrine insufficiency among diabetic patients attending MWU GRH, 2019.

\begin{tabular}{|c|c|c|c|c|c|}
\hline \multicolumn{2}{|l|}{ Independent Variables } & \multicolumn{2}{|l|}{ PEI } & \multirow[t]{2}{*}{ COR $(95 \% \mathrm{Cl})$} & \multirow[t]{2}{*}{$\mathrm{p}$-value } \\
\hline List & Category & No & Yes & & \\
\hline \multirow[t]{2}{*}{ Residence } & Urban & 145 & 24 & 1 & \multirow[t]{2}{*}{0.001} \\
\hline & Rural & 80 & 37 & $2.79(1.56,5.00)$ & \\
\hline \multirow[t]{2}{*}{ Smoking habit of the patient } & Non-smoker & 168 & 31 & 1 & \multirow[t]{2}{*}{0.000} \\
\hline & Smoker & 57 & 30 & $2.85(1.59,5.12)$ & \\
\hline \multirow[t]{2}{*}{ Alcohol drinking habit of the patient } & Never drink & 86 & 12 & 1 & \multirow[t]{2}{*}{0.000} \\
\hline & Drinker & 139 & 49 & $2.53(1.27,5.02)$ & \\
\hline \multirow[t]{2}{*}{ Insulin usage } & Yes & 72 & 33 & $2.5(1.41,4.46)$ & \multirow[t]{2}{*}{0.002} \\
\hline & No & 153 & 28 & 1 & \\
\hline \multirow[t]{2}{*}{ Hypoglycemic agent usage } & Yes & 169 & 52 & $1.92(0.89,4.13)$ & \multirow[t]{2}{*}{0.098} \\
\hline & No & 56 & 9 & 1 & \\
\hline \multirow[t]{2}{*}{ SBP } & $<140$ & 120 & 15 & 1 & \multirow[t]{2}{*}{0.000} \\
\hline & $\geq 140$ & 105 & 46 & $3.51(1.85,6.64)$ & \\
\hline \multirow[t]{2}{*}{ DBP } & $<90$ & 129 & 19 & 1 & \multirow[t]{2}{*}{0.000} \\
\hline & $\geq 90$ & 96 & 42 & $2.97(1.63,5.43)$ & \\
\hline \multirow[t]{2}{*}{ Type of DM } & Type 1 & 20 & 16 & $3.64(1.75,7.58)$ & \multirow[t]{2}{*}{0.001} \\
\hline & Type 2 & 205 & 45 & 1 & \\
\hline \multirow[t]{5}{*}{ Duration of disease } & $<5$ years & 116 & 6 & $0.03(0.01,0.10)$ & 0.000 \\
\hline & $6-10$ years & 47 & 12 & $0.15(0.05,0.43)$ & 0.000 \\
\hline & $11-15$ years & 36 & 16 & $0.27(0.10,0.74)$ & 0.011 \\
\hline & $16-20$ years & 17 & 12 & $0.42(0.14,1.28)$ & 0.129 \\
\hline & $>20$ years & 9 & 15 & 1 & 0.000 \\
\hline \multirow[t]{2}{*}{ Family history of DM } & Yes & 75 & 11 & 1 & \multirow[t]{2}{*}{0.023} \\
\hline & No & 150 & 50 & $2.27(1.12,4.62)$ & \\
\hline \multirow[t]{2}{*}{ Proteinuria } & No & 160 & 34 & 1 & \multirow[t]{2}{*}{0.014} \\
\hline & Yes & 65 & 27 & $1.96(1.09,3.50)$ & \\
\hline
\end{tabular}




\begin{tabular}{|c|c|c|c|c|c|}
\hline \multirow[t]{2}{*}{ Ketosuria } & No & 158 & 13 & 1 & \multirow[t]{2}{*}{0.000} \\
\hline & Yes & 67 & 48 & $8.71(4.43,17.12)$ & \\
\hline \multirow[t]{3}{*}{ BMI } & Normal & 99 & 30 & 1 & 0.362 \\
\hline & Overweight & 121 & 28 & $0.76(0.43,1.36)$ & 0.362 \\
\hline & Obese & 5 & 3 & $1.98(0.45,8.77)$ & 0.368 \\
\hline \multirow[t]{2}{*}{ GFR (by CKD-EPI) } & Normal & 92 & 22 & 1 & \multirow[t]{2}{*}{0.495} \\
\hline & Reduced & 133 & 39 & $1.23(0.68,2.20)$ & \\
\hline \multirow[t]{2}{*}{ Presence of AKI } & NO & 209 & 57 & 1 & \multirow[t]{2}{*}{0.880} \\
\hline & Yes & 16 & 4 & $0.92(0.30,2.85)$ & \\
\hline \multirow[t]{2}{*}{ FBS } & $<126$ & 52 & 3 & 1 & \multirow[t]{2}{*}{0.004} \\
\hline & $\geq 126$ & 173 & 58 & $5.811(1.748,19.316)$ & \\
\hline \multirow[t]{2}{*}{ BUN } & Normal & 126 & 31 & 1 & \multirow[t]{2}{*}{0.471} \\
\hline & Increased & 99 & 30 & $1.232(0.699,2.171)$ & \\
\hline \multirow[t]{2}{*}{ Creatinine } & Normal & 136 & 37 & 1 & \multirow[t]{2}{*}{0.976} \\
\hline & Increased & 89 & 24 & $0.991(0.555,1.769)$ & \\
\hline \multirow[t]{2}{*}{ Urea } & Normal & 137 & 33 & 1 & \multirow[t]{2}{*}{0.339} \\
\hline & Increased & 88 & 28 & $1.321(0.747,2.337)$ & \\
\hline \multirow[t]{2}{*}{ BUN to creatinine ratio } & $<20$ & 143 & 32 & 1 & \multirow[t]{2}{*}{0.116} \\
\hline & $\geq 20$ & 82 & 29 & $1.580(0.893,2.798)$ & \\
\hline
\end{tabular}

Single variable analysis of binary logistic regression indicated that residence, smoking habit, alcohol consumption, insulin usage, hypoglycemic agent usage, duration of the disease, type of DM, family history of DM, SBP, DBP, FBS, BUN to creatinine ratio, proteinuria and ketosuria variables were significantly associated with pancreatic exocrine insufficiency at $p$-value $<0.25$. The other variables were not significantly associated with PEI (Table 4). Variables statistically significant in single variable binary logistic regression which have $p$-value $<0.25$ were candidates for multiple logistic regression.

Diabetic patients living in rural areas were 5.86 times (AOR Cl: $2.42,14.22 \mathrm{P}$-value $=0.000$ ) more likely to suffer from pancreatic exocrine insufficiency than urban settlers. Cigarette smokers and alcohol drinkers were also 5.38 (AOR Cl: 2.10, 13.75 P-value $=0.000)$ and $3.40($ AOR Cl: 1.33, 8.65 P-value $=0.010)$ times more likely to develop PEI than non-smokers and non-alcohol drinkers respectively. 
Regarding the duration of disease, the odds of being pancreatic exocrine insufficient among diabetic patients who had diabetic duration 11 to 15 years and 16 to 20 years were $5.59(\mathrm{Cl}$ : $1.71,18.30 \mathrm{P}$-value $=$ $0.004)$ and $10.25(\mathrm{Cl}: 2.53,41.56 \mathrm{P}$-value $=0.001)$ times more likely to suffer from PEI respectively in comparison to diabetic patients below five years' duration of disease. But the there was no significant difference between of disease 6 to 10 years and under five years' duration.

Type 1 diabetics were 7.04 (AOR Cl: 1.83, 27.04 P-value = 0.005) times more likely to suffer from PEl than T2DM. Additionally, the odds of patients with ketosuria were $6.85(\mathrm{Cl}: 2.93,15.98$ P-value $=0.000)$ times more likely to suffer from PEI than those who didn't have ketosuria. Hypoglycemic agent users were more likely to develop PEl with odds ratio of $3.46(\mathrm{Cl}$ : $1.04,11.53$ P-value $=0.043)$ as compared to non-users. 
Table 5

Multivariable logistic regression analysis for Pancreatic exocrine insufficiency among diabetic patients attending MWU GRH, Bale, South East Ethiopia, 2019.

\begin{tabular}{|c|c|c|c|c|c|}
\hline \multicolumn{2}{|l|}{ Independent Variables } & \multicolumn{2}{|l|}{ PEI } & \multirow[t]{2}{*}{ AOR $(95 \% \mathrm{Cl})$} & \multirow[t]{2}{*}{ P-value } \\
\hline List & Category & No & Yes & & \\
\hline \multirow[t]{2}{*}{ Residence } & Urban & 145 & 24 & 1 & \multirow[t]{2}{*}{$0.000 * *$} \\
\hline & Rural & 80 & 37 & $5.86(2.42,14.22)$ & \\
\hline \multirow[t]{2}{*}{ Smoking habit of the patient } & Non-smoker & 168 & 31 & 1 & \multirow[t]{2}{*}{$0.000^{\star *}$} \\
\hline & Smoker & 57 & 30 & $5.38(2.10,13.75)$ & \\
\hline \multirow[t]{2}{*}{ Alcohol drinking habit of the patient } & Never drink & 86 & 12 & 1 & \multirow[t]{2}{*}{$0.010^{*}$} \\
\hline & drinker & 139 & 49 & $3.40(1.33,8.65)$ & \\
\hline \multirow[t]{2}{*}{ Hypoglycemic agent usage } & Yes & 169 & 52 & $3.46(1.04,11.53)$ & \multirow[t]{2}{*}{$0.043^{*}$} \\
\hline & No & 56 & 9 & 1 & \\
\hline \multirow[t]{2}{*}{ Type of DM } & Type 1 & 20 & 16 & $7.04(1.86,27.04)$ & \multirow[t]{2}{*}{$0.005^{\star}$} \\
\hline & Type 2 & 205 & 45 & 1 & \\
\hline \multirow[t]{5}{*}{ Duration of disease } & $<5$ years & 116 & 6 & 1 & $0.000 * *$ \\
\hline & $6-10$ years & 47 & 12 & $2.68(0.82,8.70)$ & $0.102^{\mathrm{NS}}$ \\
\hline & $11-15$ years & 36 & 16 & $5.59(1.71,18.30)$ & $0.004^{\star}$ \\
\hline & $16-20$ years & 17 & 12 & $10.25(2.53,41.56)$ & $0.001^{\star *}$ \\
\hline & $>20$ years & 9 & 15 & $26.82(2.93,117.12)$ & $0.000 * *$ \\
\hline \multirow[t]{2}{*}{ Ketosuria } & No & 158 & 13 & 1 & \multirow[t]{2}{*}{$0.000 * \star$} \\
\hline & Yes & 67 & 48 & $6.85(2.93,15.98)$ & \\
\hline \multirow[t]{2}{*}{ Family history of DM } & Yes & 75 & 11 & 1 & \multirow[t]{2}{*}{$0.562^{\mathrm{NS}}$} \\
\hline & No & 150 & 50 & $1.36(0.48,3.85)$ & \\
\hline \multirow[t]{2}{*}{ SBP } & $<140$ & 120 & 15 & 1 & \multirow[t]{2}{*}{$0.392^{\mathrm{NS}}$} \\
\hline & $\geq 140$ & 105 & 46 & $1.87(0.45,7.83)$ & \\
\hline \multirow[t]{2}{*}{ DBP } & $<90$ & 129 & 19 & 1 & \multirow[t]{2}{*}{$0.900^{\mathrm{NS}}$} \\
\hline & $\geq 90$ & 96 & 42 & $0.92(0.25,3.44)$ & \\
\hline BUN to creatinine ratio & $<20$ & 143 & 32 & 1 & $0.075^{N S}$ \\
\hline
\end{tabular}




\begin{tabular}{|c|c|c|c|c|c|}
\hline & $\geq 20$ & 82 & 29 & $2.32(0 . .92,5.85)$ & \\
\hline \multirow[t]{2}{*}{ FBS } & $<126$ & 52 & 3 & 1 & \multirow[t]{2}{*}{$0.746^{\mathrm{NS}}$} \\
\hline & $\geq 126$ & 173 & 58 & $1.29(0.28,5.85)$ & \\
\hline \multirow[t]{2}{*}{ proteinuria } & No & 160 & 34 & 1 & \multirow[t]{2}{*}{$0.230^{\mathrm{NS}}$} \\
\hline & Yes & 65 & 27 & $0.56(0.21,1.45)$ & \\
\hline \multirow[t]{2}{*}{ Insulin usage } & Yes & 72 & 33 & 1 & \multirow[t]{2}{*}{$0.823^{\mathrm{NS}}$} \\
\hline & No & 153 & 28 & $1.15(0.34,3.91)$ & \\
\hline
\end{tabular}

${ }^{*}$ ) statistically significant at $a=0.05$ with $p$-value $<0.05$

NS: statistically not significant

\section{Discussion}

The study was conducted to determine the magnitude of pancreatic exocrine impairment and associated factors among diabetic patients attending MWU GRH. The mean duration of the disease of the study participants was 11.89 (95\% Cl: 9.87, 13.91) years and $8.4(95 \% \mathrm{Cl}: 8.15,8.65)$ among T1DM and T2DM respectively. T1DM duration was in agreement with N. Ewald et al, (2009) which was 11.8 year. But, the duration of the disease among T2DM according to N. Ewald et al, (2009) observation was slightly lower than this study (7.9 years) which could be due to un-classification of individuals those whose information was not clearly registered by the researcher (16).

Both T1DM and T2DM patients can be affected by PEI. Accordingly, this study showed that the overall magnitude of pancreatic exocrine insufficiency was $21.3 \%(95 \% \mathrm{Cl}: 16.55,26.05)$ and it was higher among T1DM (44.44\%) (95\% Cl: $28.21,60.67)$ than T2DM (18.0\%) (95\% Cl: 13.24, 22.76). It may be due to absolute insulin deficiency among T1DM which resulted in the reduction of insulin trophic activity on pancreatic exocrine cells, diabetic neuropathy and autoimmunity $(5,7,17)$.

The current finding was comparable with E. Larger et al, (2012) who reported an overall PEI magnitude of $23.0 \%$, with T1DM PEl of $34.0 \%$ and T2DM of $20.0 \%$ (18). But, it was higher than the finding of M. Vujasinovic et al, (2017) that indicates overall prevalence of PEI, PEI among T1DM and PEI among T2DM with a magnitude of $5.4 \%,(6.0 \%)$ and $(5 \%)$, respectively. Such variation can be due to exclusion of diabetic patients with duration disease $<5$ years, age of patient $>70$, alcohol drinkers and the like which were the most predictors of PEI (19).

The magnitude of PEl among T2DM assessed by serum amylase and lipase $18.0 \%(95 \% \mathrm{Cl}: 13.24,22.76)$ was in line with PEI conducted by FE-1 method which was $16.8 \%$ (20). According to this study mean serum activity of amylase was decreased to $39.19 \mathrm{u} / \mathrm{l}(95 \% \mathrm{Cl}: 25.54,52.84)$ and $56.93 \mathrm{u} / \mathrm{l}(95 \% \mathrm{Cl}$ : 
$51.35,62.51)$ among T1DM and T2DM were consistent with Madole et al, (2016) also reported $32.33 \pm$ $19.99 \mathrm{u} / \mathrm{l}$ and $56.37 \pm 23.43 \mathrm{u} / \mathrm{l}$ among T1DM and T2DM, respectively. Serum lipase activity was also lowered in T1DM $28.6 \mathrm{u} / \mathrm{l}(95 \% \mathrm{Cl}$ : 16.19, 41.01) than T2DM $49.34 \mathrm{u} / \mathrm{l}(95 \% \mathrm{Cl}: 42.3,56.38)$ and similar finding was observed by Madole et al, (2016) which was $32.33 \pm 19.99 \mathrm{u} / \mathrm{l}$ among T1DM. But, mean serum activity lipase according to this study was higher than Madole et al, (2016) which was $26.13 \pm$ $13.23 \mathrm{u} / \mathrm{l}$ among T2DM and such difference may be caused by the sample size variation (21).

PEI was significantly associated with duration of disease, alcohol consumption, residence, hypoglycemic agent usage, type of DM, ketosuria and habit of cigarette smoking. Therefore, it has an effect on glycemic control and DKA management. The result of Luaces-Regueira et al, (2014) also reported that the odds of smokers were 2.4 times more likely to develop PEI significantly and alcohol consumption was not significantly associated with PEI (22). In addition to this Gonzalez et al, (2010) also reported that smokers were 1.48 times more likely to develop acute pancreatitis which may result in chronic pancreatitis (PEI) (23).

Poor glycemic control in diabetic patients may led to DKA. In current study, the odds of PEI among patients developing ketosuria was $6.85(95 \% \mathrm{Cl}: 2.93,15.98)$ times more likely than those who didn't. Even though the analysis method is not the same, Kei Nakajima \& Eiji Oda, (2017) reported ketosuria associated with low serum activity (serum amylase insufficiency) (24). In current study, diabetic patients with duration of below five years were more likely protective than those with duration of 11-15 years, 16-20 years and above 20 years. It is also supported by Ewadh et al, (2014) which reported a significant negative correlation $(-0.8)$ of amylase activity with duration of disease (11).

Type 1 diabetics were 7.04 (95\% Cl: 1.86, 27.04) more likely to suffer from PEl than T2DM. It may be due to autoimmune destruction of beta cells which led to severe exocrine cell atrophy $(8,25)$. Hypoglycemic agent users were 3.46 (95\% Cl: 1.04, 11.53) more likely to suffer from PEl than non-users. On the other hand, even though it is not statistically significant, Gonzalez et al, (2010) reported that metformin users and sulfonylurea users were more likely to develop acute pancreatitis which later on led to PEl. Such disparity might be due to study design difference and sample size inequality (23).

In current study, the odds of alcohol users were $3.40(95 \% \mathrm{Cl}: 1.33,8.65)$ times more likely to suffer from $\mathrm{PEI}$ than non-alcohol drinkers. This situation may happen due to generation of toxic metabolite and free radicals during alcohol metabolism. According to Nakajima K et al, (2011) report alcohol consumption negatively correlated with serum amylase level $(\beta$ coefficient $=-0.07)$ and significant difference between quartiles of serum amylase with alcohol consumption (26). The odds of diabetic patients living in rural area were 5.38 ( $95 \% \mathrm{Cl}: 2.42,14.22)$ times more likely to develop PEl than urban settlers. It may be due to lack access for glycemic checkup which resulted in poor glycemic control.

\section{Conclusion}

According to this study there is an evidence for the alteration of pancreatic exocrine secretion of digestive enzymes. It shows that PEI was more prevalent and more severe among T1DM than T2DM. Therefore, 
PEl has to be considered as the one of diabetic complications. About one fifth of diabetic patients suffered from pancreatic enzymes secretion derangement. The pancreatic enzymes secretion was significantly lowered by hypoglycemic agent usage, ketosuria, habit of cigarette smoking and alcohol drinking habit of the diabetic patient in the present study.

\section{Declarations}

\section{Acknowledgement}

First of all, I give thanks to my main advisor Dr. Tesaka Wondimnew and my co-advisors Mr. Gesese Bogale, Mr. Minale Fekadie and Mr. Behonegn Birhan for their invaluable support, guidance and constructive suggestions and comments for the success of this research. My Deepest and heartfelt appreciation also goes to Madda Walabu University Goba referral hospital management and clinical staffs for their cooperation during data collection and biological sample analysis.

I also give thanks to Mr. Habtamu Gezehagn, Mr. Zegeye Regassa and Mr. Damtew Solomon for their contribution in data collection, data analysis and manuscript preparation. Finally, I sincerely thank the study participants, the data collectors, MWU GRH laboratory technologists and the others those who have a contribution for the success of this research.

\section{References}

1. IDF. diabetes atlas. international diabetic federation. 2017;8.

2. Pandol SJ. Normal Pancreatic Function. Pancreapedia: The Exocrine Pancreas Knowledge Base. 2015;17.

3. Ewald N, Hardt PD. Alterations in exocrine pancreatic function in diabetes mellitus. Pancreapedia: The Exocrine Pancreas Knowledge Base. 2015;7.

4. Othman MO, Harb D, Barkin JA. Introduction and practical approach to exocrine pancreatic insufficiency for the practicing clinician. International journal of clinical practice. 2018;72(2):13066.

5. Hardt PD, Ewald N. Exocrine pancreatic insufficiency in diabetes mellitus: a complication of diabetic neuropathy or a different type of diabetes? Experimental diabetes research. 2011;10.

6. Tanvi NEJ, Akhter QS, Nahar S, Sumi MN, Hosen M. Serum amylase and lipase levels in type 2 diabetes mellitus. Journal of Bangladesh Society of Physiologist. 2017;12(2):52-6.

7. Piciucchi M, Capurso G, Archibugi L, Delle Fave MM, Capasso M, Delle Fave G. Exocrine pancreatic insufficiency in diabetic patients: prevalence, mechanisms, and treatment. International journal of endocrinology. 2015;2015.

8. Campbell-Thompson M, Rodriguez-Calvo T, Battaglia M. Abnormalities of the exocrine pancreas in type 1 diabetes. Current diabetes reports. 2015;15(10):79.

9. Chen N, Unnikrishnan R, Anjana RM, Mohan V, Pitchumoni CS. The complex exocrine-endocrine relationship and secondary diabetes in exocrine pancreatic disorders. Journal of clinical 
gastroenterology. 2011;45(10):850-61.

10. Nakajima K, Oshida H, Muneyuki T, Saito M, Hori Y, Fuchigami H, et al. Independent association between low serum amylase and non-alcoholic fatty liver disease in asymptomatic adults: a crosssectional observational study. BMJ. 2013;3(1):e002235.

11. Ewadh MJ, Juda M, Ali ZA, Ewadh MM. Evaluation of amylase activity in patients with type 2 diabetes mellitus. American Journal of Bio Science. 2014;2(5):171-74.

12. Stephanie L M Das JICK, Rinki Murphy, Anthony R J Phillips, John A Windsor, Maxim S Petrov. Relationship between the exocrine and endocrine pancreas after acute pancreatitis. World journal of gastroenterology. 2014;20(45):17196-205.

13. Guariguata L, Whiting DR, Hambleton I, Beagley J, Linnenkamp U, Shaw JE. Global estimates of diabetes prevalence for 2013 and projections for 2035. Diabetes research and clinical practice. 2014;103(2):137-49.

14. Batra H, Kumar A, Saha T, Misra P, Ambade V. Comparative study of serum amylase and lipase in acute pancreatitis patients. Indian Journal of Clinical Biochemistry. 2015;30(2):230-3.

15. WHO. World Health Organization Model List of Essential In Vitro Diagnostics Department of Essential Medicines and Health Products 2018.

16. Ewald N, Raspe A, Kaufmann C, Bretzel R, Kloer H, Hardt PJEjomr. Determinants of exocrine pancreatic function as measured by fecal elastase-1 concentrations (FEC) in patients with diabetes mellitus. 2009;14(3):118.

17. Miroslav Vujasinovic JM, Bojan Tepes. Exocrine Pancreatic Insufficiency and Diabetes Mellitus. journal of pancreas. 2016;17(3):263-8.

18. Larger E, Philippe M, Barbot-Trystram L, Radu A, Rotariu M, Nobécourt E, et al. Pancreatic exocrine function in patients with diabetes. Diabetic Medicine. 2012;29(8):1047-54.

19. Vujasinovic M, Zaletel J, Tepes B, Popic B, Makuc J, Lenart ME, et al. Low prevalence of exocrine pancreatic insufficiency in patients with diabetes mellitus. Pancreatology. 2013;13(4):343-6.

20. Terzin V, Várkonyi T, Szabolcs A, Lengyel C, Takács T, Zsóri G, et al. Prevalence of exocrine pancreatic insufficiency in type 2 diabetes mellitus with poor glycemic control. 2014;14(5):356-60.

21. Madole MB, lyer CM, Madivalar MT, Wadde SK, Howale DS. Evaluation of biochemical markers serum amylase and serum lipase for the assessment of pancreatic exocrine function in diabetes mellitus. Journal of clinical and diagnostic research: JCDR. 2016;10(11):BC01.

22. Marı 'a Luaces-Regueira P, Julio Iglesias-Garcı 'a, MD, PhD, Bjo “rn Lindkvist, MD, PhD, Margarita Castin eira-Alvarin o M, Laura Nieto-Garcl 'a, RN, Jose ' Larin o-Noia, MD,P, and J. Enrique Domı 'nguez-Mun oz M, PhD. moking as a Risk Factor for Complications in Chronic Pancreatitis. pancreas. 2014;43:275-80.

23. Gonzalez-Perez A, Schlienger RG, Rodríguez LAG. Acute pancreatitis in association with type 2 diabetes and anti-diabetic drugs: a population-based cohort study. Diabetes care. 2010;33:2580-5. 
24. Oda KNE. Ketonuria may be associated with low serum amylase independent of body weight and glucose metabolism. Journal of Metabolic Diseases. 2017.

25. Chowdhury PPCaS. A Look Inside the Pancreas: The "Endocrine-Exocrine Cross-talk". Endocrinology \& Metabolic Syndrome. 2015;4(1).

26. Nakajima K, Nemoto T, Muneyuki T, Kakei M, Fuchigami H, Munakata H. Low serum amylase in association with metabolic syndrome and diabetes: a community-based study. Cardiovascular diabetology. 2011;10(1):34.

\section{Supplementary Files}

This is a list of supplementary files associated with this preprint. Click to download.

- Annex1InformationsheetEnglishversion.pdf

- Annex2Consentform.pdf

- Annex3Questionnaire.pdf 